\title{
Embryonal rhabdomyosarcoma of the epididymis presenting as epididymitis: A case report
}

\author{
HONG-LIANG WANG ${ }^{1}$, LING-YUN LIU ${ }^{1}$, RUN-HUI TIAN $^{2}$, FU-BIAO LI $^{1}$ and KAI-MIN GUO ${ }^{1}$ \\ Departments of ${ }^{1}$ Andrology and ${ }^{2}$ Psychology, Norman Bethune First Hospital, \\ Jilin University, Changchun, Jilin 130021, P.R. China
}

Received October 19, 2015; Accepted January 13, 2016

DOI: $10.3892 / \mathrm{mco} .2016 .733$

\begin{abstract}
Paratesticular rhabdomyosarcoma (RMS) is an extremely rare malignancy in adults, accounting for $7 \%$ of all RMS cases and $6 \%$ of all non-germinal intrascrotal tumors. The clinical signs are similar to those of a hydrocele or testicular tumor, typically presenting as a unilateral, painless mass in the inguinal canal or scrotum. No specific serum markers are currently available for this tumor. RMS of the epididymis is extremely rare. Particularly when it is associated with epididymitis, this malignancy is usually overlooked. We herein present a case of epididymal embryonal RMS, manifesting an painful scrotal edema, misdiagnosed as epididymitis. The patient received 3 cycles of adjuvant chemotherapy postoperatively and remained disease-free after 4 years of follow-up.
\end{abstract}

\section{Introduction}

Rhabdomyosarcoma (RMS) is a relatively uncommon solid tumor in young patients, accounting for 4-8\% of malignant diseases in patients aged $<15$ years (1). It is reported that only $15-20 \%$ of the RMS cases arise from the spermatic cord, epididymis, testicular envelopes, prostate and bladder (2). The clinical signs of paratesticular RMS are painless masses in the inguinal canal or scrotum, displacing the testis without replacing it. We herein report the case of a patient with epididymal embryonal RMS, who presented with fever and enlargement of the right epididymis, which was misdiagnosed as epididymitis. The patient underwent 3 cycles of adjuvant chemotherapy postoperatively and remains alive and recurrence-free after 4 years of follow-up.

Correspondence to: Dr Kai-Min Guo, Department of Andrology, Norman Bethune First Hospital, Jilin University, 71 Xinmin Street, Changchun 130021, P.R. China

E-mail: 113935402@163.com

Key words: embryonal rhabdomyosarcoma, epididymis, epididymitis

\section{Case report}

A 15-year-old adolescent presented to the outpatient clinic of the Department of Andrology of the Norman Bethune First Hospital (Changchun, China) with painful edema of the scrotum on the right side that had lasted for 3 months. At the onset, the patient complained that he had experienced a mechanical injury of the scrotum while playing. The medical history of the patient was unremarkable. The physical examination revealed an enlarged, irregular, tender mass in the cauda of the epididymis, with a diameter of $5 \mathrm{~cm}$, and warm scrotal skin. The inguinal lymph nodes were not palpable bilaterally. The abdomen was soft and non-tender, without any positive findings. A scrotal ultrasound (Nemio XG 8 to $12 \mathrm{MHz}$ linear array transducer; Toshiba, Tokyo, Japan) at presentation revealed a hypoechoic swelling of the right epididymis (54x26 mm) with marked increased vascularity, suspicious for severe epididymitis. Therefore, the diagnosis of chronic epididymitis with acute onset was made and antibiotics were administered by our experienced physician.

After 3 weeks, the patient returned to the department with severe pain and scrotal swelling; he was hospitalized for further evaluation and was scheduled for an exploratory surgery. The tumor markers $\alpha$-fetoprotein, human chorionic gonadotropin and serum lactate dehydrogenase were within normal limits. During the operation, a hard, irregular tumor with no abscess was identified on the cauda of the epididymis. An intraoperative frozen-section biopsy revealed malignancy of the epididymis. A complete radical right orchiectomy via the inguinal approach and a right hemiscrotectomy were performed. An ipsilateral inguinal lymph node was also removed for biopsy. A histological examination of the surgical specimen demonstrated a spindle cell variant, cytoplasmic eosinophils with atypical nuclei and rhabdomyoblasts with eccentrically placed nuclei with prominent nucleoli (Fig. 1). The tunica albuginea had been invaded and the surgical margin was negative. On immunohistochemical examination, the tumor was found to be positive for actin and myogenin and the diagnosis of RMS was confirmed (Fig. 2). There was no evidence of metastasis on postoperative thoracic and abdominal computed tomography (CT) scans.

Following recovery, the patient received 3 cycles of adjuvant chemotherapy with the VAC regimen (vincristine $1.5 \mathrm{mg} / \mathrm{m}^{2}$, actinomycin $1.5 \mathrm{mg} / \mathrm{m}^{2}$ and cyclophosphamide $500 \mathrm{mg} / \mathrm{m}^{2}$ ), 

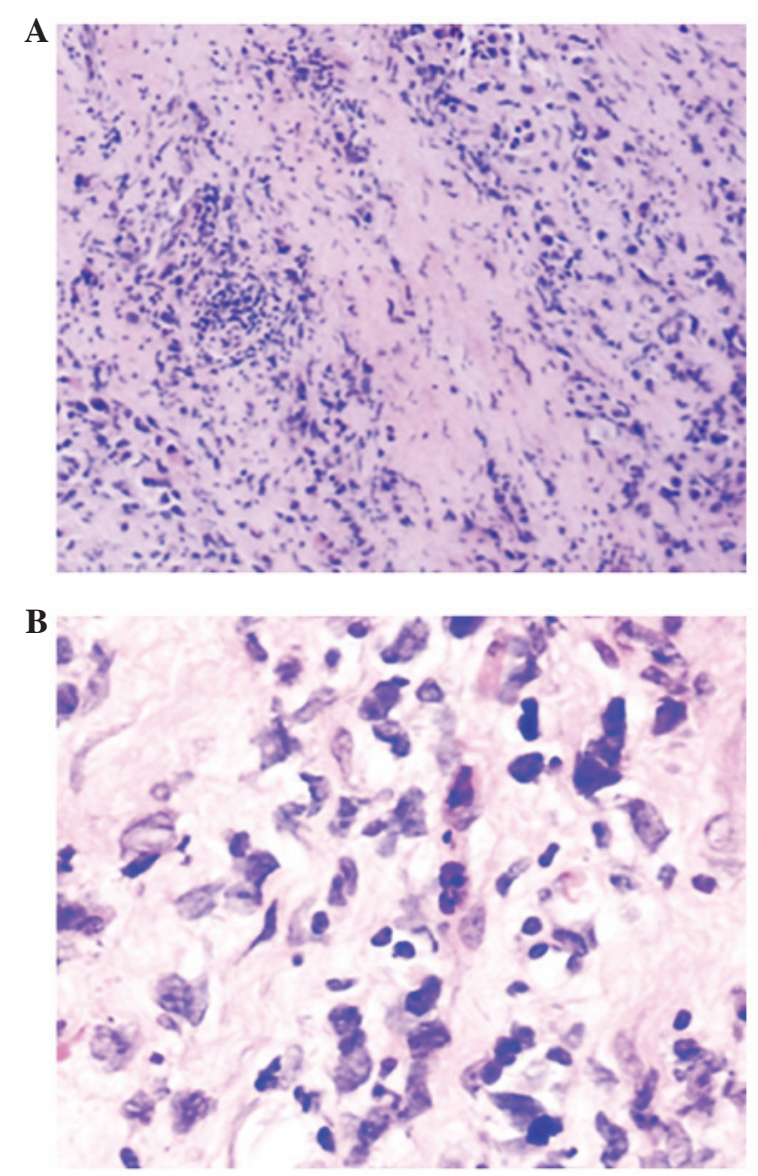

Figure 1. Hematoxylin and eosin staining of rhabdomyosarcoma. (A) Left, highly cellular anaplastic tumor; and midline, poorly cellular myxomatous tissue with ill-defined, infiltrative growth (magnification, x100) (B) Spindle cell variant, cytoplasmic eosinophils with atypical nuclei and rhabdomyoblast with eccentrically placed nuclei with prominent nucleoli (magnification, $\mathrm{x} 400$ ).

and was followed closely. After a 4-year follow-up, the patient remained in good condition, with no evidence of recurrence. No metastasis was detected during this period.

The patient and his family consented to the publication of the clinical data.

\section{Discussion}

Paratesticular RMSs, which comprise 7\% of all RMSs, are rare tumors with an aggressive growth pattern that belong to the same family of malignancies derived from primitive mesenchymal cells, such as Ewing's sarcoma, and may be related. It has been reported that the ages of 4 and 18 years represent two frequency peaks for the development of RMS (3). The most common histological types of RMS, according to the international classification of RMS, are embryonal, alveolar, botryoid embryonal, spindle cell embryonal and anaplastic (4). Our patient had an embryonal RMS, which is very rare, with only few cases reported in the literature.

The typical patient presentation for paratesticular RMS is a painless scrotal mass, or symptoms of metastasis, such as inguinal lymphadenopathy, fatigue, decreased appetite and weight loss. When patients present with a painful, edematous scrotum, they are often misdiagnosed with epididymitis.
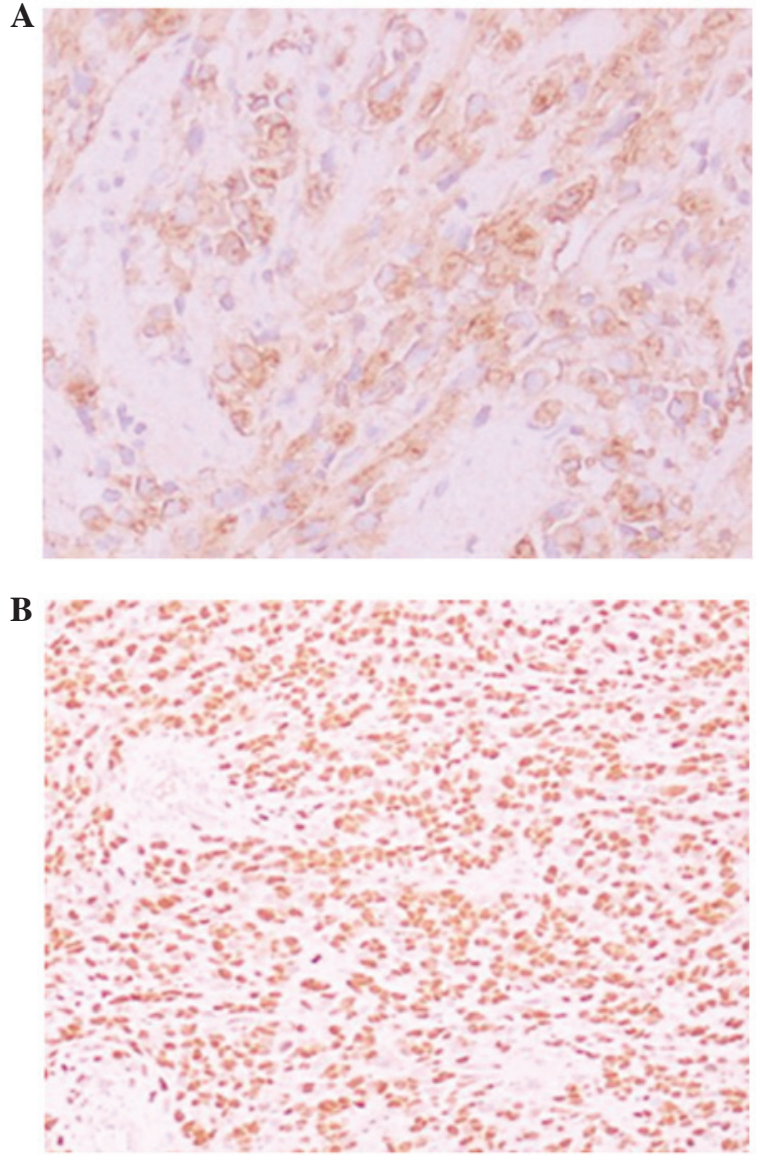

Figure 2. Immunostaining of rhabdomyosarcoma. (A) Tumor cells exhibiting cytoplasmic positivity for actin (magnification, x200); (B) nuclear positivity for myogenin (magnification, x200).

Pain has been reported in only $7 \%$ of the cases (5), whereas edema is more frequent, and a hydrocele may be occasionally present (6). Kim et al described a case of paratesticular RMS accompanied by epididymitis manifesting as painful scrotal edema (7). In our case, an edematous, painful scrotum and warm scrotal skin were the initial symptoms. Moreover, the patient recalled a history of scrotal injury. As a result, the diagnosis of malignancy was excluded. Even at the second visit, epididymitis and epididymal abscess were strongly suspected. The gradual enlargement of the testis and persistent pain, not relieved by antibiotics, prompted us to perform an exploratory surgery, during which the epididymal mass associated with epididymitis was identified. Therefore, the RMS in our patient was present concomitantly with epididymitis, which masked the primary lesion.

Scrotal sonography is the initial imaging modality for the evaluation of a scrotal mass. This imaging modality shows a mass with heterogeneous echogenicity and inguinoscrotal extension in $80 \%$ of the cases (8). Wood and Dewbury reported a case in which ultrasonography revealed increased epididymal and testicular blood flow, consistent with epididymo-orchitis (9). A thoraco-abdomino-pelvic CT scan is usually performed to evaluate lymph node and distant metastases. However, the diagnosis is confirmed using histology. The histological appearance of embryonal RMS is characterized by the presence of undifferentiated patternless spindled cells and small round blue cells. Immunohistochemical markers 
are crucial for the differential diagnosis of RMS from other primary mesenchymal and germ cell tumors also exhibiting rhabdomyoblastic differentiation (10). Markers such as myo-D1 and myoglobin are more specific compared with muscle-specific actin, smooth muscle actin and desmin, as smooth muscle markers are of limited value in differential diagnosis. The more mature muscle cells stain strongest for myoglobin, smooth muscle actin and desmin, whereas immature embryonal skeletal muscle cells stain strongest for vimentin (11). Our immunohistochemical stains were strongly and diffusely positive for actin and myogenin, suggesting a malignant mesenchymal tumor with rhabdomyoblast differentiation. Electron microscopy is considered to be of value in the diagnosis of RMS, as it demonstrates networks of myosin filaments lined by free ribosomes (12).

The management of embryonal RMS involves a multimodal approach. Complete surgical debulking followed by adjuvant chemotherapy is currently considered to be the treatment of choice. In cases who have previously undergone transscrotal surgery or if the tumor is fixed to the scrotal wall, inguinal orchiectomy and hemiscrotectomy should be performed, including radical excision of the scrotal skin (13). There has been significant controversy regarding the importance of performing a systematic lymphadenectomy, as $19-38 \%$ of the tumors present with lymph node involvement at diagnosis. Abhijith et al reported that patients aged $>10$ years, with or without radiographic evidence of retroperitoneal disease, should undergo a staging retroperitoneal lymph node dissection and receive radiation in addition to chemotherapy if the lymph nodes are positive (14). The current tendency is to avoid dissection if the radiological examinations reveal no lymph nodes sized $>1 \mathrm{~cm}$ (15). RMS is a chemosensitive tumor, and the role of multiagent chemotherapy in its treatment has been clearly demonstrated. Several chemotherapeutic protocols have been applied. The VAC, IVA and VIE protocols (E, etoposide and I, ifosfamide) were mainly used and superior results were observed with the VAC protocol (16).

The prognosis and optimal management of adult embryonic RMS is uncertain, due to its rarity. Prognosis is generally better if the tumor is confined to the scrotum and when found in younger patients, whereas adult onset of RMS, retroperitoneal location and alveolar histology are all associated with a poor prognosis (17). Combinations of polychemotherapy, surgery and radiotherapy have yielded survival rates as high as $85 \%$ in patients with localized disease (18). In the present case, visible tumor growth and tunica albuginea invasion on the final pathological examination were considered to be indications for multimodality therapy. The edema of the epididymis in our patient neither resulted in the development of embryonic RMS, nor delayed the administration of the treatment. On the contrary, this unique symptom prompted the patient to seek treatment and early intervention yielded a better prognosis.

This case was illustrative of several points worthy of notice: The clinical presentation was unusual, which made the tumor difficult to diagnose preoperatively. The location and rapidly progressive course of the tumor were typical of embryonal RMS and it may be possible to obtain successful results with chemotherapy protocols in these tumors. Considering its tendency to metastasise, long-term periodic surveillance is warranted.

\section{References}

1. Weiss AR, Lyden ER, Anderson JR, Hawkins DS, Spunt SL, Walterhouse DO, Wolden SL, Parham DM, Rodeberg DA, Kao SC and Womer RB: Histologic and clinical characteristics can guide staging evaluations for children and adolescents with rhabdomyosarcoma: A report from the Children's Oncology Group Soft Tissue Sarcoma Committee. J Clin Oncol 31: 3226-3232, 2013

2. Ahmed HU, Arya M, Muneer A, Mushtaq I and Sebire NJ: Testicular and paratesticular tumours in the prepubertal population. Lancet Oncol 11: 476-483, 2010.

3. Bouchikhi AA, Mellas S, Tazi MF, Lahlaidi K, Kharbach Y, Benhayoune K, Kanab R, Elammari JE, Khallouk A, El Fassi MJ and Farih MH: Embryonic paratesticular rhabdomyosarcoma: A case report. J Med Case Rep 7: 93, 2013.

4. Qualman S, Lynch J, Bridge J, Parham D, Teot L, Meyer W and Pappo A: Prevalence and clinical impact of anaplasia in childhood rhabdomyosarcoma: A report from the Soft Tissue Sarcoma Committee of the Children's Oncology Group. Cancer 113: 3242-3247, 2008.

5. Khoubehi B, Mishra V, Ali M, Motiwala H and Karim O: Adult paratesticular tumours. BJU Int 90: 707-715, 2002.

6. Zaslau S, Perlmutter AE, Farivar-Mohseni H, Chang WW and Kandzari SJ: Rhabdomyosarcoma of tunica vaginalis masquerading as hydrocele. Urology 65: 1001, 2005.

7. Kim YJ, Huh JS, Hyun CL and Kim SD: A case of pediatric paratesticular rhabdomyosarcoma with epididymitis. World J Mens Health 30: 146-149, 2012

8. Wiener ES, Lawrence W, Hays D, Lobe TE, Andrassy R, Donaldson S, Crist W, Newton W, Johnson J, Gehan E, et al: Retroperitoneal node biopsy in paratesticular rhabdomyosarcoma. J Pediatr Surg 29: 171-177; discussion 178, 1994.

9. Wood A and Dewbury KC: Case report: Paratesticular rhabdomyosarcoma - colour Doppler appearances. Clin Radiol 50: 130-131, 1995.

10. Kahn DG: Rhabdomyosarcoma mimicking acute leukemia in an adult: Report of a case with histologic, flow cytometric, cytogenetic, immunohistochemical, and ultrastructural studies. Arch Pathol Lab Med 122: 375-378, 1998.

11. Hardaway CA, Graham BS, Barnette DJ and Feldman BD: Embryonal rhabdomyosarcoma presenting in an adult: A case report and discussion of immunohistochemical staining. Am J Dermatopathol 25: 45-52, 2003.

12. Erlandso RA: The ultrastructural distinction between rhabdomyosarcoma and other undifferentiated 'sarcomas'. Ultrastruct Pathol 11: 83-101, 1987.

13. Chung JM, Lim YT and Lee SD: Infantile testicular rhabdomyosarcoma. Urology 69: 1208.e13-e15, 2007.

14. Abhijith SM, Nerli RB, Weiss D and Srinivasan A. Lararoscopic retroperitoneal lymph node dissection for paratesticular rhabdomyosarcoma in older children/adolescents. Indian J Surg Oncol 4: 341-344, 2013.

15. Ahsaini M, Kassogué A, Tazi MF, Idrissi KS, Moumna K and Chbani L: Early local recurrence of a pure embryonal rhabdomyosarcoma of the testis successfully managed with local resection associated with lymphadenectomy and adjuvant chemotherapy. J Afr Cancer 6: 115-118, 2014.

16. Kourda N, El Atat R, Derouiche A, Bettaib I, Baltagi S and Zermani R: Paratesticular pleomorphic rhabdomyosarcoma in an adult: Diagnosis and management. Cancer Radiother 11: 280-283, 2007 (In French).

17. Tay YK, Morelli JG, Weston WL, Stork LC and Ruyle SZ: Congenital subcutaneous nodule in an infant. Pediatr Dermatol 15: 403-405, 1998.

18. Ferrari A, Bisogno G, Casanova M, Meazza C, Piva L, Cecchetto G, Zanetti I, Pilz T, Mattke A, Treuner J and Carli M: Paratesticular rhabdomyosarcoma: Report from the Italian and German Cooperative Group. J Clin Oncol 20: 449-455, 2002. 